\title{
Estimación de la magnitud de la segregación escolar en América Latina
}

Estimating the Magnitude of School Segregation in Latin America

Estimation de la magnitude de la ségrégation scolaire en Amérique Latine

Estimativa da magnitude da segregação escoar em América Latina

Fecha de recepción: 5 DE ENERO DE 2016/Fecha de aceptación: 12 DE OCTUBRE DE 2016/Fecha de disponibilidad en línea: 20 DE FEBRERO DE 2017

Encuentre este artículo en http://magisinvestigacioneducacion.javeriana.edu.co/

(c) (i)

doi: 10.11144/Javeriana.m9-19.emse

Escrito por F. JAVIER MURILLO

UnIVERSIDAD AUTÓNOMA DE MADRID, UAM

MADRID, ESPAÑA

javier.murillo@uam.es

Cynthia Martínez-Garrido

UNIVERSIDAD DE ZARAGOZA, UNIZAR

Teruel, España

cynthia@unizar.es

\begin{abstract}
Resumen
Esta investigación busca estimar la magnitud del efecto de la segregación escolar socioeconómica, étnico-cultural y por procedencia inmigrante/nativo de los países de América Latina. Para ello, se realiza una explotación especial de la base de datos del Tercer estudio regional comparativo y explicativo, TERCE, del Laboratorio Latinoamericano de Evaluación de la Calidad de la Educación, LLECE (2013), de la Oficina Regional de Educación para América Latina y el Caribe de la Organización de las Naciones Unidas para la Educación, la Ciencia y la Cultura, OREALC/UNESCO, para estimar el índice de disimilitud a una muestra de 105.847 estudiantes de educación primaria en 5.733 escuelas de 15 países. Los resultados indican que la segregación escolar es muy alta en el conjunto de países de América Latina. La segregación escolar promedio por nivel socioeconómico es de 0,56 , de carácter étnico es de 0,92 , y por procedencia inmigrante/nativo de 0,80 .
\end{abstract}

\section{Palabras clave}

Segregación escolar; estatus socioeconómico; población indígena; inmigrante; América Latina; educación básica

\section{Transferencia a la práctica}

La presente investigación pone en la agenda educativa la grave situación de segregación escolar socioeconómica, étnicocultural y por procedencia inmigrante/nativo que se da en los países de América Latina. A partir de su cuantificación y denuncia, este estudio aporta ideas para acabar con la segregación escolar, como la dotación diferenciada de presupuesto para que las escuelas situadas en entornos más desfavorecidos cuenten con mejores recursos y medios, o el diseño de políticas educativas que garanticen la igualdad de oportunidades en el acceso a la escuela y de selección de centro.

Para citar este artículo / To cite this article / Pour citer cet article / Para citar este artigo

Murillo, F. Javier \& Martínez-Garrido, Cynthia. (2017). Estimación de la magnitud de la segregación escolar en América Latina. magis, Revista Internacional de Investigación en Educación, 9 (19), 11-30. doi: 10.11144/Javeriana.m9-19.emse 


\section{Keywords}

School segregation; socioeconomic status; indigenous population; immigrant; Latin America; basic education

\section{Abstract}

This research aims to estimate the magnitude of the effect of socioeconomic, ethnic-cultural and immigrant/native school segregation in Latin America. To this end, we collected data from the database of the Third Regional Comparative and Explanatory Study, TERCE, of the Latin American Laboratory for Evaluation of the Quality of Education, LLECE (2013), of the Regional Office of Education for Latin America and The Caribbean of the United Nations Educational, Scientific and Cultural Organization, OREALC/UNESCO, to estimate the index of dissimilarity of a sample of 105,847 students of primary education level in 5,733 schools located in 15 countries. Results indicate that school segregation is very high in Latin American countries. The average school segregation by socioeconomic level is 0.56 , by ethnic origin is 0.92 , and by immigrant/native origin is 0.80 .

\section{Transfer to practice}

This research places on the educational agenda the serious situation of socioeconomic, ethnic-cultural and immigrant/native school segregation that occurs in Latin America. Based on its quantification and denunciation, this study provides ideas for ending school segregation, such as differentiated budget allocation so that schools located in more disadvantaged areas get better resources and means, or the design of educational policies that guarantee Equal opportunities in access to school and center selection.

\section{Mots clés}

Ségrégation scolaire; statuts socioéconomique; population indigène; immigrant; Amérique Latine; éducation basique

\section{Résumé}

Cette recherche tente d'estimer la magnitude de l'effet de la ségrégation scolaire socioéconomique, ethnique-culturelle et d'origine immigrant/native des pays d'Amérique Latine. Pour cela, on réalise une exploitation spéciale de la base de données de la Troisième étude régionale comparative et explicative, TERCE, du laboratoire Latino-Américain d'évaluation de la qualité de l'éducation, LLECE (2013), du Bureau Régional de l'Education pour l'Amérique Latine et le Caraïbe de I'Organisation des Nations Unis pour l'Education, la Science et la Culture, OREALC/UNESCO, pour estimer l'index de dissimilitude à une échantillon de 105.847 étudiants d'éducation primaire en 5.733 écoles de 15 pays. Les résultats indiquent que la ségrégation scolaire moyenne par niveau socioéconomique est de 0,56 , de caractère ethnique est de 0,92 , et par origine immigrant/native de 0,80 .

\section{Transfert à la pratique}

La présente recherche installe dans l'agenda éducatif la grave situation de ségrégation scolaire socioéconomique, ethnique-culturelle et d'origine immigrant/native qui existe dans les pays d'Amérique Latine. A partir de sa quantification et l'action de porter plainte, cette étude contribue avec des idées pour finir avec la ségrégation scolaire, comme la dotation différenciée de budget pour que les écoles situées dans les entourages les plus défavorisés comptent avec les meilleures ressources et moyens, ou le dessein de politiques éducatives qui assurent l'égalité d'opportunités dans l'accès à l'école et le choix d'institutions.

\section{Palavras-chave}

Segregação escolar; status socioeconômico; população indígena; imigrante; América Latina; educação básica

\section{Resumo}

Esta pesquisa procura estimar a magnitude do efeito da segregação escolar socioeconômica, étnico-cultural e por procedência imigrante/ nativo dos países de América Latina. Para isso se realiza uma exploração especial no banco de dados do terceiro estudo regional comparativo e explicativo, TERCE, do Laboratório Latino-americano de Avaliação da Qualidade da Educação, LLECE (2013), do Escritório Regional de Educação para América Latina e o Caribe da Organização das Nações Unidas para a Educação, a Ciência e a Cultura, OREALC/UNESCO, para estimar o índice de disparidade numa mostra de 105.847 estudantes de educação primária em 5.733 escolas de 15 países. Os resultados indicam que a segregação escolar é muito alta nos conjunto países de América Latina. A segregação escolar média por nível socioeconômico é de 0,56, de caráter étnico é de 0,92, e por procedência imigrante/nativo de 0,80.

\section{Transferência à prática}

A presente pesquisa põe na agenda educativa a grave situação de segregação escolar socioeconômica, étnico-cultural e por procedência imigrante/nativo que se dá nos países da América Latina. A partir de sua quantificação e denúncia, este estudo gera ideias para acabar com a segregação escolar, como a dotação diferenciada de orçamento para que as escolas situadas em ambientes mais desfavorecidos contem com melhores recursos e meios, ou o desenho de políticas educativas que garantam a igualdade de oportunidades no acesso à escola e de seleção de centro escolar. 
No hay alternativa: solo tendremos sociedades inclusivas, cohesionadas y justas, si tenemos sistemas educativos con esas características. Pero ¿cómo son nuestros sistemas educativos?, ¿son inclusivos, cohesionados y justos?

Aunque nuestra primera reacción instintiva sea de negación, no podemos quedarnos ahí. Si queremos cambiar la realidad, necesitamos contar con evidencias empíricas válidas, fiables y actuales que aporten informaciones claras sobre la magnitud del fenómeno. El estudio de la segregación escolar nos aporta datos acerca de la inclusión, cohesión y justicia de los sistemas educativos.

El estudio de la segregación escolar, que comenzó centrándose en la segregación de carácter étnico-racial y luego se preocupó la segregación socioeconómica, tiene ya una trayectoria aproximada de medio siglo. En la actualidad, contamos con algunos datos de muchos países y conocemos la tendencia global. Sin embargo, la información que tenemos sobre la situación en América Latina es muy nueva, parcial y fragmentada. Solo muy recientemente tenemos algún estudio que, en el marco del Informe del Programa Internacional para la Evaluación de Estudiantes o Informe PISA (Programme for International Student Assessment), ha incluido varios países de la Región (Vázquez, 2012), y trabajos de países específicos: de Chile (Bellei, 2013; Valenzuela, Bellei \& de los Ríos, 2010, 2014) y de Argentina (Gasparini, Jaume, Serio \& Vázquez, 2011; Krüger, 2011, 2012, 2013).

Esta investigación busca estimar la magnitud del efecto de la segregación escolar socioeconómica, étnico-cultural y de procedencia en los países de América Latina. Para ello, se hace una explotación especial de los datos del Tercer Estudio Comparativo y Explicativo (TERCE), realizado por el Laboratorio Latinoamericano de Evaluación de la Calidad de la Educación, LLECE (2013) y publicado por la Oficina Regional de Educación para América Latina y el Caribe de la Organización de las Naciones Unidas para la Educación, la Ciencia y la Cultura, OREALC/UNESCO.

\section{Antecedentes}

En su sentido más amplio, la segregación se refiere a la distribución desigual de los individuos en diferentes estructuras jerárquicas según sus características (James \& Taeuber, 1985). Bernard Delvaux (2005), por ejemplo, habla de segregación como "la expresión de las diferencias sociales en el espacio. Aparece tan pronto como los individuos clasificados por la sociedad en distintas categorías sociales, dotadas de diferente valor social, son separados en el espacio y no hay mezclas entre unos $u$ otros" (p. 276).

Segregación escolar, por tanto, se refiere a la distribución desigual de los estudiantes en los centros educativos según sus características personales o sociales (Bellei, 2013; Dupriez, 2010; Ireson \& Hallam, 2001). Dicho en otras palabras, se da segregación escolar cuando se concentran los alumnos inmigrantes en algunas pocas escuelas, cuando los hijos de familias de menor nivel socioeconómico van a unas escuelas mientras que otras están reservadas a estudiantes con familias más pudientes; o cuando hay escuelas "de excelencia" para estudiantes con mejores resultados.

En esencia, es posible distinguir dos tipos básicos de segregación escolar: la segregación por nivel socioeconómico y la segregación étnicocultural que, a su vez, puede distinguirse entre la concentración de la población inmigrante y la de las minorías étnicas o raciales en determinadas escuelas. A estas dos hay que añadir la llamada segregación escolar por
Descripción del artículo | Article description | Description de l'article | Artigo descrição

Este artículo de investigación estima la magnitud del efecto de la segregación escolar socioeconómica, étnico-cultural y por procedencia en los países de América Latina a partir de explotación especial de la base de datos del Tercer Estudio Regional Comparativo y Explicativo, TERCE, del Laboratorio Latinoamericano de Evaluación de la Calidad de la Educación, LLECE (2013), al aplicar el índice de disimilitud a una muestra de 105.847 estudiantes en 5.733 escuelas de 15 países. Ha sido desarrollado en el marco del Proyecto I+D+i de Excelencia Escuelas en contextos socioeconómicamente desafiantes: una aproximación desde la Educación para la Justicia Social de Investigación, Referencia EDU2014-56118-P, financiado con fondos del Ministerio de Economía, Industria y Competitividad del Gobierno de España. 
capacidad, o segregación académica, que consiste en separar a los estudiantes por su nivel de competencias escolares (bien cuando el país tiene trayectorias de escolarización establecidas con base en la diferencia de nivel escolar de los estudiantes, o bien cuando los centros o la administración seleccionan a los alumnos según sus competencias); y la segregación por género, producida por la existencia de escuelas para niños y escuelas para niñas.

El estudio de la segregación escolar en todo el mundo tiene como punto de partida la decisión de la Corte Suprema de Estados Unidos sobre el caso Brown contra el Consejo de Educación de Topeka, en 19541. La misma declaró ilegal la segregación escolar por raza en ese país. A partir de ese momento y con una gran virulencia en los años 60 y 70, se multiplicaron los trabajos que buscaban estimar la magnitud de la segregación racial en Estados Unidos (Coleman, Kelly \& Moore, 1975).

Entre esos primeros estudios, quizá el más conocido es el elaborado por James Coleman, Ernest Q. Campbell, Carol J. Hobson, James McPartland, Alexander M. Mood, Frederic D. Weinfeld y Robert L. York (1966). Sus resultados evidenciaron que la existencia de escuelas para blancos y escuelas para negros agudiza la desigualdad entre grupos raciales. De igual modo, los trabajos de John R. Logan y Deirdre Oakley (2004) o Rucker C. Johnson (2011) apuntan que el nivel de segregación racial en Estados Unidos en 1968, medido por el índice de disimilitud, alcanzó valores de 0,80. Según James S. Coleman, Sara D. Kelly y John A. Moore (1975), esta cifra es algo inferior $(D=0,63)$.

Estudios recientes han aportado evidencias empíricas de que la segregación racial ha disminuido, al menos en Estados Unidos. Por ejemplo, Erica Frankenberg y Chungmei Lee (2002), utilizando el índice de exposición, encontraron que era de 0,27 en 2001, frente al 0,36 registrado en 1988. Kori J. Stroub y Meredith P. Richards (2013), por su parte, determinaron que la segregación escolar de carácter racial, usando el índice de disimilitud, había disminuido 10\% entre 1993 y 2009.

Los estudios de segregación centrados específicamente en las características socioeconómicas de las familias de los estudiantes tienen como punto de partida la década de los 80 . Uno de los trabajos más influyentes es The Truly Disadvantaged (Wilson, 1987), que estudia las desventajas de la concentración de familias de clases sociales más desfavorecidas en algunas escuelas. Desde entonces, los trabajos que abordan la segregación por nivel socioeconómico entre escuelas, su evolución o su comparación entre diferentes países se han multiplicado (Cheng \& Gorard, 2010; Dupriez, 2010; Gorard \& Smith, 2004; Jenkins, Micklewright \& Schnepf, 2008; Orfield \& Lee, 2005; Stephan, 2013).

Frente a lo encontrado con respecto a la segregación escolar étnicoracial, hay evidencias empíricas que demuestran que la magnitud de la segregación por nivel socioeconómico se está incrementando en los últimos años (Gorard, 2009; Orfield \& Lee, 2005). En Estados Unidos, Sean F. Reardon y Ann Owens (2013) señalan que la segregación escolar en las escuelas públicas de educación primaria aumentó modestamente durante los años 90 y aún más durante la primera década de los años 2000, al pasar de 0,09 a 0,11 en 2006 (utilizando el índice de raíz cuadrada). También hay evidencias de su incremento en Inglaterra (Gorard, Hordosy \& See, 2013), Bélgica (Dumay \& Dupriez, 2008), o Chile (Elaqua, 2012), entre otros países.

1 United States Supreme Court, Brown v. Board of Education of Topeka, 347 U.S. 483 (1954), Decided May 17, 1954. Disponible en: https://supreme.justia.com/cases/federal/us/347/483/ case.html 
Otros estudios analizan y comparan el nivel de segregación escolar por nivel socioeconómico entre diferentes países. Así, el trabajo realizado por Emmanuel Vázquez (2012), con datos de PISA-2009, muestra que el índice de disimilitud entre los países varía entre 0,53 y 0,26. En América Latina están los países con la mayor segregación escolar entre los estudiados (Perú: 0,53; Chile: 0,52; México: 0,49; Argentina: 0,46; Colombia: 0,46; Uruguay: 0,44, y Brasil: 0,42).

Por su parte, el trabajo elaborado por Ricard Benito-Pérez, Miguel Ángel Alegre-Canosa e Isaac González-Balletbò (2014) con los datos de 16 países de la Organización para la Cooperación y el Desarrollo Económicos, OCDE, participantes en la prueba PISA muestran que una hipotética reducción de la segregación escolar por nivel socioeconómico mejoraría la equidad en las escuelas de los países participantes.

Mucho más comunes son las investigaciones que relacionan la segregación escolar por nivel socioeconómico y la segregación étnico-cultural (Frankenberg \& Lee, 2002; Logan, Minca \& Adar, 2012; Orfield, 2001; Orfield \& Lee 2005, 2007). Estos estudios han documentado altos niveles de pobreza en determinadas escuelas, con el argumento de que los estudiantes de los grupos minoritarios están en escuelas de alta pobreza caracterizadas por contar con menos recursos y más bajos niveles de logro del estudiante (Saporito \& Sohoni, 2007).

Efectivamente, el estudio realizado por Russell W. Rumberger y Gregory J. Palardy (2005) señala que no solo hay escuelas para ricos y para pobres, sino que además esta diferenciación mantiene la diferenciación racial. De acuerdo con los autores, en las escuelas ricas, el $84 \%$ de la población es blanco, y en las escuelas pobres, el $62 \%$ de sus estudiantes pertenece a grupos minoritarios. Estos datos son confirmados por Gary Orfield y Chungmei Lee (2007) que muestran que el estudiante negro o latino promedio asiste a una escuela en la que el 60\% corresponde a población pobre; el estudiante blanco promedio asiste a una escuela en la que solo un tercio de los estudiantes es pobre.

En América Latina, la segregación escolar por nivel socioeconómico se ha abordado de forma aún muy incipiente. Destacan algunos pocos estudios como los de Gregory M. Elacqua (2012) y de Juan Pablo Valenzuela, Cristián Bellei y Danae de Los Ríos $(2010,2014)$ en Chile, y los de Leonardo C. Gasparini, David Jaume, Montserrat Serio y Emmanuel Vázquez (2011) o Natalia Krüger (2011) en Argentina.

La investigación realizada por Juan Pablo Valenzuela, Cristián Bellei y Danae de Los Ríos (2010) analiza el grado de segregación escolar por nivel socioeconómico de las escuelas en Chile. Los resultados confirman una condición de alta segregación escolar (índice de disimilitud de 0,51 en 1999 y de 0,54 en 2008). Por su parte, Leonardo C. Gasparini, David Jaume, Montserrat Serio y Emmanuel Vázquez (2011) documentan y analizan la segregación escolar desde 1986 entre los estudiantes de distintos estratos socioeconómicos que asisten a escuelas públicas y privadas en Argentina. De acuerdo con sus resultados, el grado de segregación escolar por nivel socioeconómico muestra una tendencia alcista desde mediados de los años 80 hasta la actualidad que está en valores moderados (índice de disimilitud de 0,34). Cifra, al menos, extraña.

La anterior revisión demuestra que no todos los estudios miden la segregación de la misma manera. Es más, en la actualidad, los investigadores debaten cuál es el índice utilizado para el estudio de la segregación. Los índices más conocidos para medir la segregación escolar son: el índice de aislamiento (Bell, 1954), el índice de disimilitud (Duncan \& Duncan, 1955), el índice de Stephen Gorard (2000), el índice de la raíz cuadrada (Hutchens, 
2004), o el índice de brecha por centiles (Watson, 2009). Incluso, hay algunos interesantes trabajos sobre la utilidad y ventajas de uso entre uno y otro índice (Allen \& Vignoles, 2006; Echenique \& Fryer, 2007).

De entre todos los índices, el más común entre los investigadores es el índice de disimilitud (D), también conocido como índice Duncan en referencia a sus autores. Los resultados de este índice permiten captar la dimensión de igualdad del fenómeno de segregación y reflejan la proporción de estudiantes de grupo minoritario que debería cambiar de escuela para que haya una distribución homogénea entre ellas. Su valor mínimo es el 0 y la situación de máxima segregación tiene un valor de 1. Los valores alcanzados por el índice de disimilitud se ven influenciados por la composición de la población. Se considera que la segregación es baja si $D$ toma valores entre 0 y 0,3; moderada si está entre 0,3 y 0,6; y alta cuando es superior a 0,6 (Massey \& Denton, 1994). Edward L. Glaeser y Jacob L. Vigdor (2001) hablan de hipersegregación cuando D supera el 0,6.

Como se ha visto, la investigación en América Latina adolece de falta de investigaciones globales que aborden de manera conjunta la segregación en sus escuelas y que impulsen el debate, la reflexión y la reforma de la administración y políticas educativas en la región. Por ello, en este trabajo se estima la magnitud del efecto de la segregación escolar socioeconómica, étnico-racial y por procedencia inmigrante/nativo en los países de América Latina.

\section{Metodología}

Para dar respuesta a este objetivo se realiza una explotación secundaria de los datos del Tercer Estudio Regional Comparativo y Explicativo (TERCE), del Laboratorio Latinoamericano de Evaluación de la Calidad de la Educación, LLECE (2013), de la Organización de las Naciones Unidas para la Educación, la Ciencia y la Cultura, UNESCO. El propósito del TERCE es describir qué y cuánto aprenden los estudiantes latinoamericanos de $3^{\circ} \mathrm{y}$ $6^{\circ}$ de primaria en las áreas curriculares de matemática, lengua (lectura y escritura) y ciencias naturales. En línea con otras evaluaciones internacionales como PISA, el TERCE también obtuvo información de factores asociados al aprendizaje que pudieran contextualizar y hasta cierto punto explicar los resultados académicos. Por ello, junto con las pruebas de rendimiento se aplicaron cuestionarios de contexto a estudiantes, familias y docentes que aportan los datos necesarios para este estudio.

Muestra

Una de las potencialidades del TERCE es el tamaño y la representatividad de la muestra. Concretamente, se realizó un diseño muestral estratificado, por conglomerados y bietápico (LLECE, 2013). Dado que la probabilidad de selección de escuelas en el diseño muestral propuesto es proporcional al tamaño, la probabilidad de selección de una escuela muestreada quedará definida por el indicador de tamaño propuesto, es decir, matrícula de tercero, en el caso de los alumnos seleccionados en la muestra de tercero, y la matrícula de sexto, en el caso de los alumnos seleccionados en la muestra de sexto. Para el segundo nivel de selección (aula), se selecciona aleatoriamente un aula completa, lo que implica igual probabilidad de elección para cada aula dentro de la escuela. Para el tercer nivel (alumnos), el peso muestral estará basado en una probabilidad de selección igual a uno.

Con todo ello, la muestra de estudio está compuesta por total de 105.847 estudiantes de 5.733 escuelas de 15 países latinoamericanos 
(Argentina, Brasil, Chile, Colombia, Costa Rica, Ecuador, Guatemala, Honduras, México, Nicaragua, Panamá, Paraguay, Perú, República Dominicana y Uruguay) (tabla 1).

Tabla 1

Muestra y sus características

\begin{tabular}{l|c|c|c|c|c}
\hline & $\begin{array}{l}\text { Número de } \\
\text { estudiantes }\end{array}$ & $\begin{array}{l}\text { Número de } \\
\text { escuelas }\end{array}$ & $\begin{array}{l}\text { Promedio ISECF } \\
\text { estudiantes }\end{array}$ & $\begin{array}{l}\text { Población } \\
\text { inmigrante (\%) }\end{array}$ & $\begin{array}{l}\text { Población } \\
\text { indígena (\%) }\end{array}$ \\
\hline Argentina & 5.912 & 415 & 0,43 & 1,90 & 0,00 \\
\hline Brasil & 5.384 & 302 & 0,28 & 0,00 & 0,00 \\
\hline Chile & 8.788 & 393 & 0,83 & 1,28 & 0,00 \\
\hline Colombia & 8.188 & 307 & 0,14 & 0,00 & 0,41 \\
\hline Costa Rica & 6.839 & 393 & 0,48 & 2,01 & 0,00 \\
\hline Ecuador & 9.128 & 391 & $-0,15$ & 0,00 & 0,70 \\
\hline Guatemala & 7.704 & 359 & $-0,52$ & 1,32 & 1,03 \\
\hline Honduras & 7.295 & 407 & $-0,46$ & 0,00 & 0,76 \\
\hline México & 6.453 & 336 & 0,19 & 1,84 & 0,87 \\
\hline Nicaragua & 6.749 & 380 & $-0,75$ & 0,00 & 1,07 \\
\hline Panamá & 5.945 & 372 & $-0,08$ & 4,44 & 3,03 \\
\hline Paraguay & 5.932 & 398 & 0,01 & 1,23 & 12,87 \\
\hline Perú & 9.219 & 577 & $-0,45$ & 0,00 & 5,35 \\
\hline República Dominicana & 7.082 & 365 & $-0,28$ & 0,89 & 0,00 \\
\hline Uruguay & 5229 & 338 & 0,65 & 0,00 & 0,00 \\
\hline Total/promedio & $\mathbf{1 0 5 . 8 4 7}$ & $\mathbf{5 . 7 3 3}$ & $\mathbf{0 , 0 0}$ & $\mathbf{0 , 9 9}$ & $\mathbf{1 , 7 4}$ \\
\hline \hline
\end{tabular}

Nota: ISECF: Índice socioeconómico y cultural de las familias

Fuente: elaboración propia a partir de datos del TERCE (2013)

Variables

Las variables utilizadas son de dos tipos de unidades de análisis diferenciado, de estudiante y de escuela. Las variables de estudiante son:

- Nivel socioeconómico y cultural de la familia del estudiante (ISECF): factor obtenido a partir de los niveles educativos y ocupacionales de los padres, volumen de ingresos económicos familiares, características de la vivienda y disponibilidad de libros en el hogar. Índice estandarizado para la región. Variable tipificada. Los gráficos 1 y 2 representan la distribución del nivel socioeconómico y cultural de las familias de la muestra y la media para los diferentes países.

- Pertenencia de la familia a grupo indígena. Variable dummy a partir del autoinforme de los padres.

- Procedencia de la familia: nativo-inmigrante. Variable dummy obtenida también a partir del autoinforme de los padres.

Estas tres variables se obtuvieron a partir del cuestionario para las familias. Este instrumento, que consta de 37 preguntas cerradas con varias alternativas de respuesta, aporta información acerca de las características socioeconómicas, demográficas y culturales de las familias. 
Además, para delimitar los resultados, se tuvo en consideración el hábitat de la escuela, es decir, si el centro docente donde estudian los alumnos está en una zona urbana o rural. Variable dummy, esta información procede de los criterios de muestreo utilizados en el TERCE.

\section{Análisis de datos}

Para estimar la magnitud del efecto de la segregación escolar en los diferentes países de América Latina se utiliza el índice de disimilitud (D) o índice de Duncan, que considera la proporción de estudiantes del grupo minoritario en las escuelas respecto del grupo mayoritario, y se interpreta como la tasa de estudiantes que deben cambiar de escuela para que la segregación sea nula.

Para estimar la magnitud de la segregación socioeconómica, en este estudio se considera el grupo minoritario a partir de dos criterios diferentes. Por un lado, los estudiantes pertenecientes al Q1 (el 25\% inferior) en índice socioeconómico y cultural de las familias (ISECF) para cada país; y el grupo mayoritario el resto de estudiantes. Con ello, tenemos una aproximación del grado de segregación de los estudiantes más vulnerables. Por otra parte, el grupo minoritario es el de los estudiantes pertenecientes al Q4 en ISECF, es decir, el 25\% cuyas familias tienen un mayor nivel socioeconómico. Con ello, se analiza la segregación de los estudiantes con más recursos. Esta doble mirada es una novedad metodológica de esta investigación, y aporta una perspectiva más global del fenómeno.

Para calcular la segregación étnico-racial, el grupo minoritario está formado por los estudiantes pertenecientes a un grupo indígena, y para estudiar la segregación escolar por procedencia, el grupo minoritario está formado por los estudiantes cuyas familias sean inmigrantes.

En el índice de disimilitud, la máxima segregación se alcanza cuando el índice toma valor 1, y la mínima cuando toma valor 0 . La fórmula estándar para estimar D es la siguiente:

$$
D=\frac{1}{2} \sum_{i=1}^{k}\left|\frac{x_{1 i}}{X_{1}}-\frac{x_{2 i}}{X_{2}}\right|
$$

Donde, para cada país, $\mathrm{x}_{1 \mathrm{i}}=$ número de estudiantes del grupo minoritario (estudiantes cuartil 1 de ICSEF del país, o de familias indígenas, o inmigrantes) en la escuela i $\mathrm{x}_{2 \mathrm{i}}=$ número de estudiantes del grupo mayoritario (del cuartil 2, 3 o 4 en ISECF del país, o no indígena, o nativo) en la escuela i
$\mathrm{X}_{1}$ y $\mathrm{X}_{2}=$ número total de estudiantes minoritarios y mayoritarios, respectivamente, en todas las escuelas del país.

\section{Resultados}

En primer lugar, se presentan los resultados de estimar la magnitud de la segregación socioeconómica en los países de América Latina, para seguir con la segregación étnico-racial, y la segregación por procedencia de las familias de los estudiantes nativos o inmigrantes.

\section{Segregación escolar socioeconómica}

Uno de los desafíos de los sistemas educativos de América Latina es la alta segregación de sus escuelas por cuestiones socioeconómicas. El promedio del índice de segregación escolar socioeconómica de los países de América Latina es de 0,56, cifra que puede ser considerada casi de hipersegregación. Más concretamente, la segregación escolar del grupo más vulnerable llega a 0,54; mientras que la segregación de los alumnos con más recursos es del 0,58.

Estas cifras globales esconden una importante variabilidad entre países. Así, Panamá, Honduras y Perú son globalmente los tres países con mayor segregación escolar de la región; República Dominicana, Costa Rica, Guatemala y Uruguay registran una menor segregación global. También cabe destacar que, salvo Panamá y México, en todos los países la segregación es mayor con los estudiantes ubicados en Q4 en ISECF que en el Q1.

De esta forma, es posible configurar tres grupos de países en función de la magnitud de su índice de segregación:

1. Países con una segregación media-alta (con un índice de disimilitud de hasta 0,5): República Dominicana $(0,43)$ y Costa Rica $(0,49)$.

2. Países con una segregación alta (entre 0,5 y $0,6)$ : Uruguay $(0,52)$, Argentina $(0,53)$, Guatemala $(0,54)$, Brasil y Chile $(0,55)$, Nicaragua $(0,56)$, Ecuador y Paraguay (cada uno con 0,58$)$, y México y Colombia (cada uno con 0,59$)$.

3. Países con una segregación escolar por razones socioeconómicas muy alta (más de 0,60): Perú (con 0,60), Honduras $(0,62)$ y Panamá $(0,63)$.

Los países con una mayor diferencia entre la segregación de los grupos más y menos desfavorecidos son Colombia y Chile con 0,1 puntos de diferencia, y Ecuador, con 0,09 puntos. 
Tabla 2

Segregación escolar socioeconómica en países de América Latina. Índice de disimilitud para Q1 y Q4 en ISECF como grupos minoritarios y promedio, por país. Total de centros

\begin{tabular}{|c|c|c|c|}
\hline & $\begin{array}{l}\text { Índice D } \\
\text { con Q1 } \\
\text { como grupo } \\
\text { minoritario }\end{array}$ & $\begin{array}{l}\text { Índice D } \\
\text { con Q4 } \\
\text { como grupo } \\
\text { minoritario }\end{array}$ & $\begin{array}{l}\text { Índice D } \\
\text { medio }\end{array}$ \\
\hline Argentina & 0,5214 & 0,5338 & 0,5276 \\
\hline Brasil & 0,5262 & 0,5672 & 0,5467 \\
\hline Chile & 0,4982 & 0,5960 & 0,5471 \\
\hline Colombia & 0,5294 & 0,6559 & 0,5927 \\
\hline Costa Rica & 0,4596 & 0,5155 & 0,4875 \\
\hline Ecuador & 0,5356 & 0,6257 & 0,5807 \\
\hline Guatemala & 0,5067 & 0,5662 & 0,5364 \\
\hline Honduras & 0,5985 & 0,6470 & 0,6228 \\
\hline México & 0,5880 & 0,5840 & 0,5860 \\
\hline Nicaragua & 0,5627 & 0,5632 & 0,5629 \\
\hline Panamá & 0,6727 & 0,5965 & 0,6346 \\
\hline Paraguay & 0,5610 & 0,5937 & 0,5773 \\
\hline Perú & 0,5886 & 0,6146 & 0,6016 \\
\hline República Dominicana & 0,3956 & 0,4736 & 0,4346 \\
\hline Uruguay & 0,4922 & 0,5559 & 0,5240 \\
\hline Promedio países & 0,5358 & 0,5793 & 0,5575 \\
\hline
\end{tabular}

Fuente: elaboración propia a partir de datos del TERCE (2013)

Estas cifras pueden entenderse mejor al analizar el porcentaje de escuelas que concentran más estudiantes cuyas familias están entre el 25\% con un menor índice socioeconómico (tabla 3). Así, el país con menor segregación es también el que tiene un menor porcentaje de escuelas que concentran muchos estudiantes del Q1. Efectivamente, República Dominicana tiene solo el 2,23\% con más del 75\% de estudiantes del Q1 y el 8,38\% con más del $50 \%$.

Un razonamiento contrario puede hacerse con los países más segregados de la región. En Panamá, el 13,17\% de las escuelas tiene más del 75\% de los estudiantes del Q1, cinco puntos más que el promedio de los países participantes en el estudio; y en Perú, el 35,18\% de las escuelas tiene más del $50 \%$ de los estudiantes de familias con menos recursos. La concentración de estudiantes más vulnerables en algunas escuelas es más que evidente.

El análisis del porcentaje de escuelas que concentran más estudiantes de mayor nivel socioeconómico nos da una imagen complementaria de la 
segregación. En la tabla 3 puede observarse que Colombia, México y Chile son los tres países con más segregación de estudiantes con familias en el Q4, y Argentina el país con una menor segregación de este tipo.

Tabla 3

Porcentaje de centros con más del 75 \% y del 50\% de estudiantes de Q1 y Q4 en ISECF. Total de centros

\begin{tabular}{|c|c|c|c|c|}
\hline & $\begin{array}{l}\text { Centros } \\
\text { con más del } \\
75 \% \text { de los } \\
\text { alumnos } \\
\text { del Q1 }(\%)\end{array}$ & $\begin{array}{l}\text { Centros } \\
\text { con más del } \\
50 \% \text { de los } \\
\text { alumnos } \\
\text { del Q1 }(\%)\end{array}$ & $\begin{array}{l}\text { Centros } \\
\text { con más del } \\
75 \% \text { de los } \\
\text { alumnos } \\
\text { del Q4 }(\%)\end{array}$ & $\begin{array}{l}\text { Centros } \\
\text { con más del } \\
50 \% \text { de los } \\
\text { alumnos } \\
\text { del Q4 (\%) }\end{array}$ \\
\hline Argentina & 14,86 & 30,48 & 3,74 & 11,22 \\
\hline Brasil & 6,90 & 20,69 & 10,76 & 20,49 \\
\hline Chile & 10,16 & 22,40 & 21,19 & 27,91 \\
\hline Colombia & 9,93 & 27,48 & 22,00 & 29,33 \\
\hline Costa Rica & 6,99 & 17,88 & 11,98 & 16,93 \\
\hline Ecuador & 7,89 & 15,53 & 12,86 & 22,31 \\
\hline Guatemala & 1,67 & 12,26 & 11,27 & 18,59 \\
\hline Honduras & 8,68 & 19,11 & 20,20 & 27,93 \\
\hline México & 12,31 & 22,22 & 20,72 & 30,03 \\
\hline Nicaragua & 6,97 & 20,11 & 8,20 & 14,55 \\
\hline Panamá & 13,17 & 21,01 & 14,97 & 22,88 \\
\hline Paraguay & 4,65 & 19,90 & 11,69 & 22,08 \\
\hline Perú & 16,07 & 35,18 & 11,50 & 17,88 \\
\hline República Dominicana & 2,23 & 8,38 & 10,34 & 21,23 \\
\hline Uruguay & 4,83 & 18,73 & 6,53 & 15,13 \\
\hline Promedio países & 8,49 & 20,76 & 13,20 & 21,23 \\
\hline
\end{tabular}

Fuente: elaboración propia a partir de datos del TERCE (2013)

Una de las causas más importantes de la segregación escolar es la segregación residencial. En los lugares donde solo hay un centro educativo, este necesariamente recoge a todos los alumnos de esa zona. Si allí hay una alta concentración de familias de determinado nivel socioeconómico, la segregación será alta. Dado que esta situación se produce esencialmente en las zonas rurales, es interesante estudiar la segregación que se da en las zonas urbanas.

En la tabla 4 se muestran los índices de segregación para cada país, considerando únicamente los centros situados en zonas urbanas. Con esta restricción, la anterior situación se matiza, aunque las tendencias se mantienen. De hecho, el promedio del índice de segregación escolar socioeconómica considerando solo los centros situados en entornos urbanos es igual a los índices para la totalidad de los centros: de 0,54 para los centros urbanos, 0,56 para el total. Esta disminución se debe a la bajada en el índice de segregación escolar considerando como grupo minoritario el Q1 de los estudiantes según su ISECF, que baja de 0,54 a 0,47. 
En esta situación, los países con una menor segregación escolar socioeconómica global siguen siendo República Dominicana $(0,43)$ y Costa Rica $(0,48)$, a los que se suma Argentina $(0,50)$. Y el país con una mayor segregación escolar continúa siendo Panamá $(0,62)$, al que se suma Guatemala $(0,61)$.

Es interesante, en todo caso, verificar que en todos los países el índice de segregación en las escuelas urbanas considerando como grupo minoritario el Q4 es mayor que considerando el Q1. En Colombia y Guatemala, esta cifra es considerablemente más alta en el Q4 (hasta 0,24 puntos y 0,23 puntos, respectivamente), y en Costa Rica, Argentina y Uruguay es ligeramente mayor (tabla 4).

Tabla 4

Segregación escolar socioeconómica en escuelas urbanas en países de América Latina. Índice de disimilitud para Q1 y Q4 en ISECF como grupos minoritarios y promedio, por país

\begin{tabular}{|c|c|c|c|}
\hline & $\begin{array}{l}\text { Índice D con } \\
\text { Q1 como grupo } \\
\text { minoritario }\end{array}$ & $\begin{array}{l}\text { Índice D con } \\
\text { Q4 como grupo } \\
\text { minoritario }\end{array}$ & $\begin{array}{l}\text { Índice D } \\
\text { medio }\end{array}$ \\
\hline Argentina & 0,4698 & 0,5350 & 0,5024 \\
\hline Brasil & 0,4668 & 0,5625 & 0,5146 \\
\hline Chile & 0,4412 & 0,5911 & 0,5162 \\
\hline Colombia & 0,4219 & 0,6644 & 0,5432 \\
\hline Costa Rica & 0,4486 & 0,5134 & 0,4810 \\
\hline Ecuador & 0,4920 & 0,6328 & 0,5624 \\
\hline Guatemala & 0,4960 & 0,7245 & 0,6102 \\
\hline Honduras & 0,4897 & 0,6463 & 0,5680 \\
\hline México & 0,4537 & 0,5764 & 0,5151 \\
\hline Nicaragua & 0,5062 & 0,6270 & 0,5666 \\
\hline Panamá & 0,5632 & 0,6847 & 0,6240 \\
\hline Paraguay & 0,4673 & 0,6433 & 0,5553 \\
\hline Perú & 0,4707 & 0,6079 & 0,5393 \\
\hline República Dominicana & 0,3741 & 0,4858 & 0,4300 \\
\hline Uruguay & 0,4819 & 0,5567 & 0,5193 \\
\hline Promedio países & 0,4696 & 0,6035 & 0,5365 \\
\hline
\end{tabular}

Fuente: elaboración propia a partir de datos del TERCE (2013)

Un análisis, análogo al anterior, del porcentaje de centros que agrupa estudiantes en el Q1 y Q4 en ISECF (con menores y mayores recursos), también deja una imagen similar a la que teníamos con la totalidad de los centros, con la gran diferencia de que, al haber menos estudiantes del Q1, hay muchos menos centros donde se concentran estos niños y niñas (tabla 5). 
Tabla 5

Porcentaje de centros urbanos con más del 75\% y del 50\% de estudiantes de Q1 y Q4 en ISECF

\begin{tabular}{|c|c|c|c|c|}
\hline & $\begin{array}{l}\text { Centros con más } \\
\text { del } 75 \% \text { de los } \\
\text { alumnos del Q1 }(\%)\end{array}$ & $\begin{array}{l}\text { Centros con más } \\
\text { del } 50 \% \text { de los } \\
\text { alumnos del Q1 (\%) }\end{array}$ & $\begin{array}{l}\text { Centros con más } \\
\text { del } 75 \% \text { de los } \\
\text { alumnos del Q4 (\%) }\end{array}$ & $\begin{array}{l}\text { Centros con más } \\
\text { del } 50 \% \text { de los } \\
\text { alumnos del Q4 (\%) }\end{array}$ \\
\hline Argentina & 2,83 & 11,79 & 6,05 & 19,53 \\
\hline Brasil & 0,95 & 7,11 & 14,90 & 28,37 \\
\hline Chile & 0,39 & 6,23 & 29,84 & 39,53 \\
\hline Colombia & 0,60 & 3,57 & 37,20 & 50,61 \\
\hline Costa Rica & 4,83 & 13,90 & 14,11 & 19,94 \\
\hline Ecuador & 3,53 & 9,02 & 14,96 & 28,74 \\
\hline Guatemala & 0,00 & 0,86 & 23,68 & 32,46 \\
\hline Honduras & 0,00 & 0,00 & 54,55 & 71,33 \\
\hline México & 0,48 & 2,90 & 32,68 & 47,80 \\
\hline Nicaragua & 0,00 &, 00 & 25,20 & 39,02 \\
\hline Panamá & 0,00 & ,71 & 39,26 & 59,26 \\
\hline Paraguay & 0,53 & 1,60 & 23,50 & 44,26 \\
\hline Perú & 0,69 & 5,50 & 22,81 & 35,44 \\
\hline República Dominicana & 0,00 & 3,98 & 16,52 & 32,59 \\
\hline Uruguay & 1,72 & 15,02 & 9,24 & 21,43 \\
\hline Total & 1,10 & 5,48 & 24,30 & 38,02 \\
\hline
\end{tabular}

Fuente: elaboración propia a partir de datos del TERCE

Segregación étnico-racial

El análisis por segregación étnico-racial, como se señaló, monopolizó la preocupación de los investigadores estadounidenses sobre segregación escolar en los años 60 y 70, pero sigue presente en la actualidad. En ese caso, el interés se centraba en la segregación de los afroamericanos y, recientemente, de los latinos en Estados Unidos. En América Latina, sin embargo, el interés se focaliza en la segregación escolar de los estudiantes de familias pertenecientes a la población indígena.

Para este estudio, el TERCE aporta información sobre la pertenencia de los padres a un grupo indígena según su propia descripción. Y sobre esta realidad realizaremos el estudio de segregación escolar étnica. Ello implica que posiblemente esté medido de una forma muy conservadora. Sea por ello o por azares del muestreo, en seis países no hay ningún estudiante con padres pertenecientes a un grupo indígena. Entre ellos, Brasil o Chile.

Igual que hicimos para la segregación socioeconómica, presentamos los datos para el conjunto de las escuelas y solo para las escuelas urbanas. Aunque en este caso, la interpretación es diferente. Los datos se presentan en la tabla 6.

La segregación escolar étnica en América Latina es extremadamente alta, con un promedio en los nueve países con alumnos con familias de población indígena (y para todas las escuelas) de 0,92. Cifra muy próxima al 1 que es la máxima segregación. Este 0,92 significa que el 92\% de los estudiantes de grupos étnicos minoritarios deberían cambiar de escuela para conseguir la desegregación perfecta. 
El análisis por países muestra que ocho de los nueve con estudiantes del grupo étnico originario tienen índice de segregación de más de 0,9; es decir, extremadamente alto, en Colombia a 0,996 y en México a 0,987. La única excepción es Paraguay, donde la segregación es muy alta, pero anota un 0,63 muy lejos de las cifras del resto de países.

Si consideramos solo las escuelas situadas en contextos urbanos, las cifras son muy parecidas. El promedio para el conjunto de América Latina es de 0,89 , prácticamente igual que para el total de las escuelas. El estudio de cada país muestra cifras análogas a las encontradas para el conjunto de las escuelas: más de 0,9 para todos los países, excepto en Paraguay que es de 0,57. Aunque llama la atención Perú, que pasa de 0,91 para el conjunto de las escuelas a 0,92 para las escuelas urbanas.

Tabla 6

Segregación escolar étnico-racial en países de América Latina. Índice de disimilitud. Total centros y centros urbanos

\begin{tabular}{|c|c|c|}
\hline & Total centros & Centros urbanos \\
\hline Argentina & - & - \\
\hline Brasil & - & - \\
\hline Chile & - & - \\
\hline Colombia & 0,9957 & - \\
\hline Costa Rica & - & - \\
\hline Ecuador & 0,9630 & 0,9480 \\
\hline Guatemala & 0,9031 & 0,9068 \\
\hline Honduras & 0,9666 & 0,9233 \\
\hline México & 0,9866 & 0,9669 \\
\hline Nicaragua & 0,9783 & 0,9602 \\
\hline Panamá & 0,9346 & 0,9203 \\
\hline Paraguay & 0,6291 & 0,5731 \\
\hline Perú & 0,9088 & 0,9209 \\
\hline República Dominicana & - & - \\
\hline Uruguay & - & - \\
\hline Promedio países & 0,9184 & 0,8899 \\
\hline
\end{tabular}

Fuente: elaboración propia a partir de datos del TERCE

Segregación por procedencia inmigrante/nativo

Aunque el fenómeno de la inmigración tiene magnitud y características diferentes a las de otros países donde se ha estudiado más, en especial en Europa, es interesante conocer hasta qué punto hay segregación escolar hacia el colectivo de estudiantes de padres inmigrantes.

De nuevo en este caso, la consideración acerca de si un niño de una familia inmigrante ha sido muy conservadora: se ha considerado exclusivamente así solo cuando los padres así lo han afirmado. Eso genera, una vez más, que en siete de los 15 países no haya ningún estudiante de familia inmigrante en la muestra de estudio. Es el caso de Brasil, Colombia, Ecuador, Honduras, Nicaragua, Perú y Uruguay. Y en los países que sí hay, 
estos lo son en una proporción muy baja. Esta postura tan conservadora, en todo caso, no altera los resultados del estudio de segregación, sino que lo hacen más fiable.

El promedio de la segregación escolar por procedencia inmigrante/ nativo en los países de América Latina es de 0,80 (tabla 7). Cifra muy alta. Recordemos que Edward L. Glaeser y Jacob L. Vigdor (2001) sostienen que hay hipersegregación cuando este índice supera 0,6.

En el análisis por países la muestra presenta una gran uniformidad en los índices encontrados. Todos ellos superan ese 0,6 con creces. Pero también con importantes diferencias. Desde Paraguay, que tiene la máxima segregación $(0,92)$ a Panamá con la menor, aunque también muy alta $(0,65)$. En todo caso hay que considerar, como luego abordaremos, que la inmigración en esos dos países tiene características muy diferentes.

Al estimar los índices de segregación considerando solo los centros urbanos, encontramos, de nuevo, una gran estabilidad de los resultados; se da en esencia la misma segregación para todos los centros. Ello es, entre otras cosas, una clara prueba de la fiabilidad de los resultados.

Con ello, podemos establecer tres grupos de países en función de la magnitud de su segregación escolar por procedencia:

- Países con una segregación extrema, de más de 0,85 en índice de disimilitud: Paraguay y República Dominicana.

- Países con una segregación muy muy alta (entre 0,80 y 0,85): Argentina y Chile.

- Países con una segregación escolar por procedencia bastante alta, menos de 0,80: Guatemala, México, Costa Rica y Panamá.

Tabla 7

Segregación escolar según el origen nativo o inmigrante de los estudiantes en América Latina. Índice de disimilitud. Total centros y centros urbanos

\begin{tabular}{|c|c|c|}
\hline & Total centros & Centros urbanos \\
\hline Argentina & 0,8353 & 0,8271 \\
\hline Brasil & - & - \\
\hline Chile & 0,8202 & 0,8021 \\
\hline Colombia & - & - \\
\hline Costa Rica & 0,7687 & 0,7646 \\
\hline Ecuador & - & - \\
\hline Guatemala & 0,7878 & 0,7528 \\
\hline Honduras & - & - \\
\hline México & 0,7805 & 0,7840 \\
\hline Nicaragua & - & - \\
\hline Panamá & 0,6458 & 0,6907 \\
\hline Paraguay & 0,9178 & 0,8933 \\
\hline Perú & - & - \\
\hline República Dominicana & 0,8502 & 0,8618 \\
\hline Uruguay & - & - \\
\hline Promedio países & 0,8008 & 0,7970 \\
\hline
\end{tabular}

Fuente: elaboración propia a partir de datos del TERCE (2013) 
Una mirada conjunta de los resultados obtenidos indica que la segregación escolar socioeconómica, étnico-racial y por procedencia constituye fenómenos diferentes. En la tabla 8 se observa que hay poca relación entre la inmigración y el nivel socioeconómico, en el sentido de que el 16,5\% de los estudiantes de familias inmigrantes pertenece al $25 \%$ con mayor nivel socioeconómico. Esas cifras, sin embargo, son muy dispares entre unos países y otros. En Argentina, solo el 4,7\% de los estudiantes de familias inmigrantes está entre el 75\% de mayor ICSEF (Q4), mientras que el porcentaje en Paraguay es del 71,6\% y en República Dominicana del 22,5\%. Eso quiere decir que la segregación escolar por procedencia tiene características muy diferenciadas con otras regiones del mundo, por ejemplo, con Europa. Mientras que en Europa está muy asociada a la segregación socioeconómica, en América Latina, en especial en algunos países, tiene características muy diferentes.

La relación entre pertenencia a población indígena y situación socioeconómica, por su parte, no es tan independiente, pero tampoco es posible afirmar que hay una relación absoluta. Aunque, de nuevo, la diferencia entre países es muy importante. En Colombia el 98,6\% de los estudiantes con familias pertenecientes a población indígena está en el Q1 de ISECF, en Nicaragua solo está el 12,5\%.

Tabla 8

Relación entre nivel socioeconómico (ICSEF) y pertenencia a población indígena y a población inmigrante para el conjunto de países de América Latina

\begin{tabular}{l|c|c}
\hline & $\begin{array}{l}\text { Estudiantes } \\
\text { con familias } \\
\text { de población } \\
\text { inmigrante (\%) }\end{array}$ & $\begin{array}{l}\text { Estudiantes } \\
\text { con familias } \\
\text { de población } \\
\text { indígena (\%) }\end{array}$ \\
\hline Estudiantes en el Q1 en ISECF & 35,54 & 62,87 \\
\hline Estudiantes en el Q2 en ISECF & 29,18 & 28,18 \\
\hline Estudiantes en el Q3 en ISECF & 21,67 & 7,68 \\
\hline Estudiantes en el Q4 en ISECF & 13,62 & 1,28 \\
\hline Total & 100,00 & 100,00 \\
\hline \hline
\end{tabular}

Nota: Datos ponderados para el conjunto de América Latina

Fuente: elaboración propia a partir de datos del TERCE (2013)

\section{Discusión}

En este artículo se han aportado cifras sobre la situación actual, con datos válidos y fiables, de la magnitud de segregación escolar en los países de América Latina, tanto socioeconómica, como étnico-cultural, o por procedencia inmigrante/nativo. Y la situación encontrada ha sido de muy alta segregación en todos y cada uno de los 15 países estudiados.

Pero antes de presentar un diálogo entre los resultados encontrados en este estudio con otros de objetivos análogos, es preciso señalar que los resultados de índices de segregación diferentes no son automáticamente comparables entre sí, pero muestran la existencia de tendencias intrapaíses e interpaíses. En esta investigación, hemos elegido el índice de disimilitud, frente a otros como el de la raíz cuadrada o el de Stephen Gorard, por ser el más habitual en este tipo de trabajos y por ofrecernos una imagen, por tanto, más comprensiva. La novedad ha sido usarlo en un doble sentido, no solo con el Q1 como grupo minoritario, sino también con 
el Q4. Esta decisión amplía la mirada y abre las puertas a una mayor comprensión del fenómeno.

Los resultados obtenidos respecto a la segregación escolar socioeconómica son coherentes con los encontrados en las pocas investigaciones realizadas en la región. El estudio realizado en Chile por los profesores Juan Pablo Valenzuela, Cristián Bellei y Danae de Los Ríos (2010), seguramente el mejor de los hechos en ese país hasta el momento, aporta un índice de disimilitud de 0,54 en 2008 y aumentando en el tiempo. Esta cifra es absolutamente acorde con el 0,56 para el Q1 encontrado en este trabajo. En Argentina destaca el trabajo de Leonardo C. Gasparini, David Jaume, Montserrat Serio y Emmanuel Vázquez (2011), y aunque insiste en la idea de que estos índices están creciendo, sus datos de 0,34 en 2008 distan mucho de los nuestros. Ellos usan como "grupo minoritario" el primer quintil y en esta investigación el primer cuartil, pero ello no explica en absoluto esas disparidades.

Aunque quizá el estudio más comparable es el realizado por Emmanuel Vázquez (2012) que usa otra base de datos procedente de una evaluación internacional, en este caso, el Informe PISA, pero con datos un poco anteriores: 2008. Como señalamos, se encontró que este índice estaba en torno al 0,5. Aunque son algunas centésimas más bajos que los nuestros, y salvo alguna excepción, sí coinciden en señalar el orden de los países, pues Perú tiene la más alta segregación y Uruguay la más baja. En cualquier caso, se muestra que los países de América Latina son los que presentan una mayor segregación de entre los países del mundo sobre los que hay datos.

La falta de estudios sobre segregación escolar étnico-racial y por inmigración realizados en América Latina, hace que sea más difícil el diálogo con trabajos previos. Aunque también es cierto que el escaso número de familias que se han autoconsiderado como de grupo indígena o inmigrante hace que estos resultados haya que tomarlos con más precaución.

\section{Conclusiones}

América Latina es la región más inequitativa del mundo (PNUD, 2010) y sus sistemas educativos reflejan y reproducen esa desigualdad. Este estudio ha demostrado, con datos recientes y con una amplia muestra, la existencia de una alta segregación escolar en todos y cada uno de los países de la región, segregación socioeconómica, étnico-racial y entre los estudiantes inmigrantes. Superar este problema es, sin duda, uno de los mayores desafíos de la región en la construcción de sociedades más inclusivas, cohesionadas y justas.

Los tres tipos de segregaciones escolares estudiadas tienen diferentes características. Mientras que la segregación de los grupos indígenas tiene sus ele- mentos positivos por lo que supone de respeto a su cultura y ante la necesidad de una escuela más adaptada a su idiosincrasia, la segregación socioeconómica y de los estudiantes inmigrantes supone un lastre para una escuela y una sociedad mejor.

Multitud de estudios relaciona la segregación escolar con el desarrollo académico de los estudiantes y la tasa de abandono (Driessen, 2002; Felouzis, 2003), pero también con el desarrollo socioafectivo de los estudiantes (Agirdag, Van Avermaet \& Van Houtte, 2013). De forma que si se quiere mejorar el desarrollo de todos los estudiantes, es imprescindible abordar el tema de la segregación escolar.

Un tema clave es que en América Latina, las escuelas donde se concentran los estudiantes más vulnerables son también las que tienen peores instalaciones e infraestructuras. F. Javier Murillo-Torrecilla y Marcela Román (2011) recordaban que en América Latina el $10 \%$ de las escuelas no cuenta con servicios básicos como agua corriente o luz eléctrica, y que ese hecho está directamente asociado con el desarrollo de los estudiantes. Además, tienen los docentes y directivos con una peor preparación (Murillo-Torrecilla \& Martínez-Garrido, 2015), lo que sin duda multiplica los efectos negativos de la segregación escolar.

De ahí que una de las medidas más importantes para limitar los efectos negativos de la segregación escolar es hacer un esfuerzo por dotar estas escuelas situadas en entornos más desfavorecidos con mayor presupuesto, mejores instalaciones y medios; se trata incluso de una política para atraer a los mejores docentes y directivos hacia estas escuelas. Si estamos convencidos de que la escuela debe dar un apoyo especial a aquellos que por sus condiciones más lo necesitan, es justo pensar en la necesidad de que los gobiernos den un mayor apoyo a aquellas escuelas que tienen mayores dificultades. Como decía John Rawls (1971), no es suficiente con que las instituciones básicas de la sociedad sean ordenadas y eficientes, es necesario que sean justas. Y un sistema educativo justo es aquel que dé un trato diferencial en función de las necesidades.

Paro ello, quizá no es suficiente apostar por regulaciones en el acceso y la distribución escolar, como el caso de las políticas de zonificación para atajar la segregación. Como señala Miguel Ángel AlegreCanosa (2010), el desafío sería entonces que estas políticas fueran capaces de producir un marco en el que todas las familias tuvieran las mismas opciones reales de acceder a una diversidad significativa de escuelas, que todos tuvieran las mismas limitaciones a la hora de escoger su centro educativo de preferencia.

Este estudio tiene como primera virtud el uso de una base de datos con información amplia y actual, que confiere a sus resultados un interés notable. En este estudio se utilizan datos de más de 100.000 
alumnos y 5.000 escuelas, recogidos con un muestreo de calidad lo que hace que tengan una alta fiabilidad. Sin embargo, no podemos dejar de destacar dos cuestiones. En primer lugar, el pequeño número de estudiantes por escuela: unos 20 estudiantes en promedio. En este sentido, sería deseable que el cuarto estudio del Laboratorio Latinoamericano de Evaluación de la Calidad de la Educación, LLECE, tomara datos de más aulas por escuela. El coste añadido sería mínimo y las ventajas muchas. La otra limitación, ya comentada, es que se ha optado por utilizar los autoinformes de los padres y madres respecto a la pertenencia en un grupo indígena y procedencia de otro país. Eso ha hecho que el número de estudiantes en esa situación sea muy pequeño. No está tan claro en qué sentido esta decisión afecta los resultados. En todo caso, es el primer estudio realizado con estas características y con esta visión tan global, lo que le hace tener un interés inequívoco.

Una idea conclusiva que se extrae de este trabajo es la necesidad de incrementar los estudios e investigaciones sobre la segregación escolar en los diferentes países de América Latina y el Caribe. Tal como empezamos este artículo, solo si tenemos evidencias de un hecho es posible tomar las medidas adecuadas para cambiarlo.

Hemos visto que América Latina tiene unos índices de segregación escolar muy altos, inaceptables, posiblemente los más altos del mundo. Si queremos sociedades más igualitarias, más justas e inclusivas, tenemos que empezar por hacer nuestros sistemas educativos más justos e inclusivos. No hay atajos.

\section{Sobre los autores}

F. Javier Murillo es profesor de la Universidad Autónoma de Madrid, UAM, y director del doctorado en educación. Es coordinador de la Red Iberoamericana de Investigación sobre Cambio y Eficacia Escolar, RINACE. Autor de más de un centenar de publicaciones sobre calidad, eficacia y mejora de la educación. Más información en www. uam.es/javier.murillo

Cynthia Martínez-Garrido es profesora en la Universidad de Zaragoza, UNIZAR. Doctora en ciencias de la educación, Universidad Autónoma de Madrid, UAM. Es editora de REICE. Revista Iberoamericana sobre Calidad, Eficacia y Cambio en Educación, y coeditora de RIEJS, Revista Internacional de Educación para la Justicia Social. Más información en www.cmartinezgarrido.es

\section{Referencias}

Agirdag, Orhan; Avermaet, Piet van \& Houtte, Mieke van (2013). School Segregation and Math Achievement: A Mixed-Method Study on the Role of Self-Fulfilling Prophecies. Teachers College Record, 115 (3), 1-50. Disponible en: http://citeseerx.ist.psu.edu/viewdoc/download? doi=10.1.1.706.5666\&rep $=$ rep1\&type $=$ pdf

Alegre-Canosa, Miguel Ángel (2010). Casi-mercados, segregación escolar y desigualdad educativa: una trilogía con final abierto. Educação \& Sociedade, 31 (113), 1157-1178. Disponible en: http://www.scielo.br/ pdf/es/v31n113/06.pdf

Allen, Rebecca \& Vignoles, Anna (2006). What Should an Index of School Segregation Measure? London: London School of Economics, Centre for the Economics of Education, CEE. Disponible en: http://cee.Ise. ac.uk/ceedps/ceedp60.pdf

Bell, Wendell (1954). A Probability Model for the Measurement of Ecological Segregation. Social Forces, 32 (4), 357-364. Disponible en: https://www. 
researchgate.net/publication/245000687_A Probability_Model_for_the_Measurement_of_Ecological_Segregation

Bellei, Cristián (2013). El estudio de la segregación socioeconómica y académica de la educación chilena. Estudios Pedagógicos, 39 (1), 325-345. Disponible en: http://www.scielo.cl/pdf/estped/ v39n1/art19.pdf

Benito-Pérez, Ricard; Alegre-Canosa, Miguel Ángel \& González-Balletbò, Isaac (2014). School Segregation and its Effects on Educational Equality and Efficiency in 16 OECD Comprehensive School Systems. Comparative Education Review, 58 (1), 104-134.

Cheng, Shou Chen \& Gorard, Stephen (2010). Segregation by Poverty in Secondary Schools in England 2005-2009: A Research Note. Journal of Education Policy, 25 (3), 415-418.

Coleman, James S.; Campbell, Ernest Q.; Hobson, Carol J.; McPartland, James; Mood, Alexander M.; Weinfeld, Frederic D. \& York, Robert L. (1966). Equality of Educational Opportunity. Washington, DC: US Government Printing Office. Disponible en: http://files.eric.ed.gov/fulltext/ ED012275.pdf

Coleman, James S.; Kelly, Sara D. \& Moore, John A. (1975). Trends in School Segregation, 1968-73. Washington, DC: The Urban Institute. Disponible en: http://files.eric.ed.gov/fulltext/ED117252.pdf

Delvaux, Bernard (2005). Ségrégation scolaire dans un contexte de libre choix et de ségrégation résidentielle. En Marc Demeuse, Ariane Baye, MarieHélène Straeten, Julien Nicaise \& Anne Matoul (eds.). Vers une école juste et efficace: 26 contributions sur les systèmes d'enseignement et de formation: une approche internationale, 275296. Bruxelles: De Boeck Université.

Driessen, Geert (2002). School Composition and Achievement in Primary Education: A Largescale Multilevel Approach. Studies in Educational Evaluation, 28 (4), 347-368. Disponible en: http://www. geertdriessen.nl/wp-content/uploads/2014/11/ see02-driessen-schoolcomposition.pdf

Dumay, Xavier \& Dupriez, Vincent (2008). Does the School Composition Effect Matter? Evidence from Belgian Data. British Journal of Educational Studies, 56 (4), 440-477. Disponible en https://www.researchgate.net/ publication/229698422_Does_the_school_ composition_effect_matter_Evidence_from _ Belgian_data

Duncan, Otis Dudley \& Duncan, Beverly (1955). A Methodological Analysis of Segregation Indexes. American Sociological Review, 20 (2), 210-217. Disponible en: http://personal.psc.isr.
umich.edu/yuxie-web/files/demtech/Duncan_ Duncan1955.pdf

Dupriez, Vincent (2010). Methods of Grouping Learners at School. Paris: United Nations Educational, Scientific and Cultural Organization, UNESCO. Disponible en: http://unesdoc.unesco. org/images/0018/001876/187616e.pdf

Echenique, Federico \& Fryer, Roland G., Jr. (2007). A Measure of Segregation Based on Social Interactions. The Quarterly Journal of Economics, 122 (2), 441-485. Texto disponible en: http://scholar.harvard.edu/files/fryer/files/a_measure_of segregation_based_on_social_interactions.pdf

Elacqua, Gregory M. (2012). The Impact of School Choice and Public Policy on Segregation: Evidence from Chile. International Journal of Educational Development, 32 (3), 444-453.

Felouzis, Georges (2003). La ségrégation ethnique au collège et ses conséquences. Revue Française de Sociologie, 44 (3), 413-447. Disponible en: http://www.cairn.info/article.php?ID_ARTICLE= RFS_443_0413\&Docld $=205588$

Frankenberg, Erica \& Lee, Chungmei (2002). Race in American Public Schools: Rapidly Resegregating School Districts. Boston, MA: Harvard Civil Rights Project. Disponible en: https://www.civilrights project.ucla.edu/research/k-12-education/inte gration-and-diversity/race-in-american-publicschools-rapidly-resegregating-school-districts/ frankenberg-rapidly-resegregating-2002.pdf

Gasparini, Leonardo C.; Jaume, David; Serio, Montserrat \& Vázquez, Emmanuel (2011). La segregación escolar en Argentina. Reconstruyendo la evidencia. Buenos Aires: Centro de Estudios Distributivos, Laborales y Sociales, CEDLAS. Disponible en: http://cedlas.econo.unlp.edu.ar/archi vos_upload/doc_cedlas123.pdf

Glaeser, Edward L. \& Vigdor, Jacob L. (2001). Racial Segregation in the 2000 Census: Promising News. Washington, DC: The Brookings Institution. Disponible en: https://www.brookings.edu/ research/racial-segregation-in-the-2000-cen sus-promising-news/, https://www.brookings. edu/wp-content/uploads/2016/06/glaeser.pdf

Gorard, Stephen (2000). Education and Social Justice: the Changing Composition of Schools and its Implications. Cardiff: University of Wales Press.

Gorard, Stephen (2009). Does the Index of Segregation Matter? The Composition of Secondary Schools in England since 1996. British Educational Research Journal, BERJ, 35 (4), 639-652. Disponible en: http://eprints.bham.ac.uk/303/

Gorard, Stephen; Hordosy, Rita \& See, Beng Huat (2013). Narrowing Down the Determinants of between-School Segregation: An Analysis of the 
Intake to All Schools in England, 1989-2011. Journal of School Choice: International Research and Reform, 7 (2), 182-195.

Gorard, Stephen \& Smith, Emma (2004). An International Comparison of Equity in Education Systems. Comparative Education, 40 (1), 15-28.

Hutchens, Robert (2004). One Measure of Segregation. International Economic Review, 45 (2), 555-578.

Ireson, Judith \& Hallam, Susan (2001). Ability Grouping in Education. London: Paul Chapman Publishing.

James, David R. \& Taeuber, Karl E. (1985). Measures of Segregation. En Nancy B. Tuma (ed.). Sociological Methodology, 1-32. San Francisco, California: Jossey-Bass.

Jenkins, Stephen P.; Micklewright, John \& Schnepf, Sylke V. (2008). Social Segregation in Secondary Schools: How Does England Compare with Other Countries? Oxford Review of Education, 34 (1), 21-37.

Johnson, Rucker C. (2011). Long-Run Impacts of School Desegregation \& School Quality on Adult Attainments. National Bureau of Economic Research, NBER Working Paper Series, Working Paper 16664. Disponible en: http://socrates.berkeley.edu/ ruckerj/johnson_schooldese gregation_NBERw16664.pdf

Krüger, Natalia (2011). The Segmentation of the Argentine Education System: Evidence from PISA 2009. Regional and Sectoral Economic Studies, 11 (3), 41-64. Disponible en: http://www.usc.es/economet/ journals2/eers/eers1133.pdf

Krüger, Natalia (2012). Equidad educativa interna y externa en Argentina: un análisis para las últimas décadas (Tesis de doctorado en Economía). Universidad Nacional del Sur, Bahía Blanca, Argentina. Disponible en: http://repositoriodigital.uns.edu.ar/bitstream/123456789/2424/1/ Tesis\%20doctoral\%20Kr\%C3\%BCger.pdf

Krüger, Natalia (2013). Segregación social y desigualdad de logros educativos en Argentina. Education Policy Analysis Archives/Archivos Analíticos de Políticas Educativas, 21 (86), 1-26. Disponible en: http://epaa. asu.edu/ojs/article/download/1352/1418

Laboratorio Latinoamericano de Evaluación de la Calidad de la Educación, LLECE (2013). Tercer estudio regional comparativo y explicativo, TERCE. Santiago: Oficina Regional de Educación para América Latina y el Caribe de la Organización de las Naciones Unidas para la Educación, la Ciencia y la Cultura, OREALC/UNESCO. Disponible en: http://www. unesco.org/new/es/santiago/education/education-assessment/thirdregional-comparative-and-explanatory-study-terce/

Logan, John R.; Minca, Elisabeta \& Adar, Sinem (2012). The Geography of Inequality: Why Separate Means Unequal in American Public Schools. Sociology of Education, 85, 287-301. Disponible en: https:// www.ncbi.nlm.nih.gov/pmc/articles/PMC3832260/

Logan, John R. \& Oakley, Deirdre (2004). The Continuing Legacy of the Brown Decision: Court Action and School Segregation, 1960-2000. Nueva York: Lewis Mumford Center for Comparative Urban and Regional Research. Disponible en: https://s4.ad.brown.edu/Projects/ usschools/reports/report2.pdf

Massey, Douglas S. \& Denton, Nancy A. (1994). Hypersegregation in U.S. Metropolitan Areas: Black and Hispanic Segregation along Five Dimensions. Demography, 26 (3), 373-393.

Murillo-Torrecilla, F. Javier \& Martínez-Garrido, Cynthia (2015). La formación de directores y directoras, un factor (más) de inequidad escolar en América Latina. Revista Iberoamericana de Educación, 69, 241266. Disponible en: http://rieoei.org/rie69a11.pdf 
Murillo-Torrecilla, F. Javier \& Román, Marcela (2011). School Infrastructure and Resources Do Matter: Analysis of the Incidence of School Resources on the Performance of Latin American Students. School Effectiveness and School Improvement, 22 (1), 29-50.

Orfield, Gary (2001). Schools More Separate: Consequences of a Decade of Resegregation. Boston, Massachusetts: Harvard University Press. Disponible en: http://files.eric.ed.gov/fulltext/ ED459217.pdf

Orfield, Gary \& Lee, Chungmei (2005). Why Segregation Matters: Poverty and Educational Inequality. Boston, Massachusetts: Harvard University Press. Disponible en: https://www.civilrightsproject. ucla.edu/research/k-12-education/integrationand-diversity/why-segregation-matters-povertyand-educational-inequality/orfield-why-segre gation-matters-2005.pdf

Orfield, G. \& Lee, Chungmei (2007). Historic Reversals, Accelerating Resegregation, and the Need for New Integration Strategies. Boston, Massachusetts: Harvard University Press. Disponible en: https://www.civilrightsproject.ucla.edu/ research/k-12-education/integration-and-diver sity/historic-reversals-accelerating-resegrega tion-and-the-need-for-new-integration-strate gies-1/orfield-historic-reversals-accelerating.pdf

Programa de las Naciones Unidas para el Desarrollo, PNUD (2010). Informe Regional sobre Desarrollo Humano para América Latina y el Caribe 2010. Actuar sobre el futuro: romper la transmisión intergeneracional de la desigualdad. Nueva York: Programa de las Naciones Unidas para el Desarrollo, PNUD. Disponible en: http://hdr.undp.org/ sites/default/files/rhdr-2010-rblac.pdf

Rawls, John (1971). A Theory of Justice. Boston, Massachusetts: Harvard University Press.

Reardon, Sean F. \& Owens, Ann (2013). 60 Years after Brown [Supreme Court's 1954 Brown v. Board of Education decision]: Trends and Consequences of School Segregation. Annual Review of Sociology, 40, 199-218.

Rumberger, Russell W. \& Palardy, Gregory J. (2005). Does Segregation Still Matter? The Impact of
Student Composition on Academic Achievement in High School. The Teachers College Record, 107 (9), 1999-2045. Disponible en: http:// intranet.niacc.edu/pres_copy(1)/ILC/Does\%20 Segregation\%20Still\%20Matter\%20-The\%20 Impact\%20of\%20Student\%20Composition\%20 on\%20Academic\%20Achievement\%20in\%20 High\%20School.pdf

Saporito, Salvatore \& Sohoni, Deenesh (2007). Mapping Educational Inequality: Concentrations of Poverty among Poor and Minority Students in Public Schools. Social Forces, 85 (3), 1227-1254.

Stephan, Walter (ed.) (2013). School Desegregation: Past, Present, and Future. Nueva York: Springer.

Stroub, Kori J. \& Richards, Meredith P. (2013). From Resegregation to Reintegration: Trends in the Racial/Ethnic Segregation of Metropolitan Public Schools, 1993-2009. American Educational Research Journal, 50 (3), 497-531.

Valenzuela, Juan Pablo; Bellei, Cristián \& Los Ríos, Danae de (2010). Segregación escolar en Chile. En Sergio Martinic \& Gregory Elacqua (eds.). ¿Fin de ciclo? Cambios en la gobernanza del sistema educativo chileno, 209-229. Santiago: UNESCO. Disponible en: http://unesdoc.unesco.org/ images/0019/001905/190544S.pdf

Valenzuela, Juan Pablo; Bellei, Cristián \& Los Ríos, Danae de (2014). Socioeconomic School Segregation in a Market-Oriented Educational System. The Case of Chile. Journal of Education Policy, 29 (2), 217-241.

Vázquez, Emmanuel (2012). Segregación escolar por nivel socioeconómico. Midiendo el fenómeno $y$ explorando sus determinantes. Buenos Aires: Centro de Estudios Distributivos, Laborales y Sociales, CEDLAS. Disponible en: http://cedlas.eco no.unlp.edu.ar/download.php? file=archivos upload/doc_cedlas128.pdf

Watson, Tara (2009). Inequality and the Measurement of Residential Segregation by Income in American Neighborhoods. Review of Income and Wealth, ROIW, 55 (3), 820-844. Disponible en: http://www.roiw.org/2009/2009-25.pdf

Wilson, William Julius (1987). The Truly Disadvantaged. Chicago, Illinois: University of Chicago Press. 